\title{
Biclique edge-choosability in some classes of graphs*
}

\author{
Gabriel A. G. Sobral ${ }^{1}$, Marina Groshaus ${ }^{2}$, André L. P. Guedes ${ }^{1}$ \\ ${ }^{1}$ Department of Informatics - Federal University of Paraná (UFPR) \\ Curitiba - PR - Brasil \\ ${ }^{2}$ CONICET \\ Buenos Aires, Argentina \\ \{gagsobral, andre\}@inf.ufpr.br, marinagroshaus@yahoo.es
}

\begin{abstract}
In this paper we study the problem of coloring the edges of a graph for any $k$-list assignment such that there is no maximal monochromatic biclique, in other words, the $k$-biclique edge-choosability problem. We prove that the $K_{3}$ free graphs that are not odd cycles are 2-star edge-choosable, chordal bipartite graphs are 2-biclique edge-choosable and we present a lower bound for the biclique choice index of power of cycles and power of paths. We also provide polynomial algorithms to compute a 2-biclique (star) edge-coloring for $K_{3}$-free and chordal bipartite graphs for any given 2-list assignment to edges.
\end{abstract}

\section{Introduction}

There are many problems related to coloring graphs. In this work we define an edgecoloring problem called the the $k$-biclique edge-choosability problem. We call a biclique an induced complete bipartite subgraph and if a maximal biclique is colored only with one color then we say it's a maximal monochromatic biclique.

The first related problem was studied by Pablo Terlisky in his master thesis in 2010 [Terlisky 2010]. He defined the $k$-biclique coloring analogously to the $k$-clique coloring problem, studied by Dániel Marx [Marx 2011], as coloring the vertices of a graph with $k$ colors such that there is no maximal monochromatic biclique. A biclique can be defined as a induced maximal complete bipartite subgraph. Terlisky proved that the $k$-biclique coloring problem is $\Sigma_{2}^{\mathcal{P}}$-complete in general case for $k \geq 2$. Furthermore Hélio B. Macedo Filho et al. [Figueiredo et al. 2013] showed that to verify if a power of a cycle or a power of a path is biclique colorable is $\operatorname{coN} \mathcal{P}$-complete.

Also, the $k$-biclique coloring problem was studied in the context of list coloring by Marina Groshaus et al. in 2012 [Soulignac et al. 2012]. They showed that $k$-biclique choosability and $k$-star choosability are $\Pi_{3}^{\mathcal{P}}$-complete for $k>2$ in general case. A power of a path $P_{n}^{k}$ is graph with $n$ vertices which $\left\{v_{i}, v_{j}\right\} \in E\left(P_{n}^{k}\right)$ if and only if $|i-j| \leq k$. And a power of a cycle $C_{n}^{k}$ is a graph with $n$ vertices which $\left\{v_{i}, v_{j}\right\} \in E\left(C_{n}^{k}\right)$ if and only if $\min \{(j-i) \bmod n,(i-j) \bmod n\} \leq k$. Note that a $P_{n}^{k}$ is a subgraph of $C_{n}^{k}$.

When focus on coloring edges of the graph, the natural $k$-biclique edge-coloring problem arises. It was defined by Dantas et al. as coloring the edges of a graph with $k$ colors such that there is no maximal monochromatic biclique [Guedes et al. 2017]. They proved that the 2-biclique edge-coloring problem is $\mathcal{N} \mathcal{P}$-Hard in the general case, $K_{3}$-free

\footnotetext{
*Partially supported by CAPES/MathAmSud 021/14, ANPCyT PICT-2013-2205 and CONICET.
} 
graphs that are not odd cycles are 2-star edge-colorable, chordal bipartite are 2-biclique edge-colorable and power of paths and power of cycles are biclique edge-colorable using at most 4 colors. A graph is chordal bipartite if there is a perfect edge without vertex elimination ordering, which each removed edge induces a biclique.

A natural question that arises is what happens when Dantas et al. results are considered in the context of list coloring. Given a graph $G$ and a list color assignment $L$ to its edges, $G$ is $L$-biclique edge-colorable if each edge of $G$ can be colored using one color from its list such that the (non trivial) bicliques of $G$ are not monochromatic. The $k$-biclique edge-choosability problem is to decide if a given graph $G$ is $L$-biclique edgecolorable for every list color assignment $L$ with $k$ colors to each edge. The biclique choice index of a graph $G\left(c_{B}^{\prime}(G)\right)$ is the minimum $k$ such that $G$ is $k$-biclique edge-choosable. As the star is specific case of biclique then the $k$-star edge-choosability problem can be defined analogously just replacing biclique by star. Since a graph choice index is at least equals to its chromatic index [Rubin et al. 1979], then it is important to check the relationship of graph biclique chromatic index and its biclique choice index. Therefore the objective of this paper is to extend Dantas et al. results to list coloring context by studying the $k$-biclique edge-choosability problem.

We prove that the $K_{3}$-free graphs that are not odd cycles are 2-star edge-choosable, chordal bipartite are 2-biclique edge-choosable and present a lower bound for the biclique choice index of power of cycles and power of paths.

\section{Star edge-choosability problem in $K_{3}$-free graphs}

In this section we are going to show that $K_{3}$-free graphs not isomorphic to odd cycles, are 2-star edge-choosable and provide a polynomial time algorithm to compute a 2-star edge-coloring. The odd cycles are forbidden since in this case a proper edge coloring is equivalent to color the edges of the graph such that there is no maximal monochromatic star $\left(K_{1, r}\right)$. However odd cycles are not 2-edge-colorable then they are not 2-star edgechoosable.

Theorem 1. The $K_{3}$-free graphs not isomorphic to odd cycles, are 2-star edge-choosable.

Proof. Let $G$ be a connected (otherwise use each connected component) $K_{3}$-free graph not isomorphic to any odd cycle. If $G$ is an even cycle then coloring its edges is equivalent to color the stars of $G$. As even cycles are 2-choosable [Rubin et al. 1979] then $G$ is 2star edge-choosable. Otherwise, $G$ is not an even cycle, then we show that $G$ is 2 -star edge-choosable by giving an algorithm for coloring the edges of the graph.

If there is a vertex $v$ with degree 1 , consider a depth first search (DFS) of $G$ started at $v$. In that way there are no return edges to the root $v$. If there is no vertex of degree 1 , then there is a cycle $C=\left(v_{1}, \ldots, v_{\ell}\right)$. Assuming $G$ is not a cycle, there is a vertex of $C$ that has a neighbor, $w$, outside the $C$. Suppose w.l.o.g. that $v_{\ell}$ is that vertex. Consider a DFS started at $v_{1}$ such that $v_{2}, \ldots, v_{\ell}$ appear in that sequence and before $w$. In that case, there is at least a returning edge $\left\{v_{\ell}, v_{1}\right\}$ (the root) and $v_{\ell}$ is not a leaf of the DFS.

We color the tree edges with a color different from the edge that is above in the tree, since the list has size 2 this assignment is always possible. After that, the stars centered at vertex that are not the root or a leaf of the DFS are already not monochromatic. 
If the root has degree 1 , its star is trivial. Otherwise, if the star centered at the root is still monochromatic, color the return edge $\left\{v_{\ell}, v_{1}\right\}$ with a different color. For the stars centered at leaves of the DFS with return edges, choose one of its return edges and color with a different color. Every non trivial star is not monochromatic. The remaining return edges are colored with any color since all the stars are already colored with at least two colors. We assure that every maximal star has two colors and then $G$ is 2-star edge-choosable.

Since it is a DFS, the algorithm runs in polynomial time.

\section{Biclique edge-choosability in chordal bipartite graphs}

In this section we prove that every chordal bipartite graph is 2-biclique edge-choosable and we also give a polynomial time algorithm to compute a 2-biclique edge-coloring.

Theorem 2. Every chordal bipartite graph is 2-biclique edge-choosable.

Proof. We are going to show that every chordal bipartite graph $G$ is 2-biclique edgechoosable by assigning inductively a color to an edge $e_{i}$ using its perfect edge-withoutvertex elimination ordering $\left(e_{1}, \ldots, e_{m}\right)$ [Kratsch and Kloks 1995]. The base case is when $|E(G)|=2$ and in this case it is always possible to set a different color to each of the edges. By inductive hypothesis we have that $G_{i-1} \backslash e_{i}$ is 2-biclique edge-choosable. As $e_{i}$ is bisimplicial then $e_{i}$ belongs only to one biclique, so it is enough to set a color to $e_{i}$ different from the edges of the biclique which contains $e_{i}$, if it is still monochromatic. And since the order $\left(e_{1}, \ldots, e_{m}\right)$ traverses all bicliques of $G$, then when a color is set to $e_{1}$ there is no monochromatic biclique for any 2-list assignment and thus $G$ is 2-biclique edge-choosable.

As the naive method to find a bisimplicial edge (the vertices of an edge induces a complete bipartite graph) takes $O\left(|E|^{2}\right)$ steps then the algorithm to compute a 2-biclique edge-coloring is $O\left(|E|^{3}\right)$.

\section{Biclique edge-choosability in power of paths and power of cycles}

In this section we are going to present a lower bound for the biclique choice index of power of cycles and power of paths, that is, the $c h_{B}^{\prime}\left(C_{n}^{k}\right)$ is the maximum between 2 and a natural $\alpha>0$. We remark that the power of paths are sub-graphs of power of cycles when we remove the edges from the last $k$ vertices to the first $k$ vertices of the graph.

Theorem 3. The biclique choice index $\operatorname{ch}_{B}^{\prime}\left(C_{n}^{k}\right)>\max \{2, \alpha\}$, if $k \geq 2, n>4 k$ and $\left\lceil\frac{k}{2}\right\rceil \geq\left(\begin{array}{c}2 \alpha-1 \\ \alpha\end{array}\right)$.

Proof. Let $H$ be the a graph defined by $V(H)=\left\{v_{i j} \mid\left\{v_{i}, v_{j}\right\} \in E\left(C_{n}^{k}\right)\right\}$ and $E(H)=$ $\left\{\left\{v_{i j}, v_{j l}\right\} \mid\left\{v_{i}, v_{l}\right\} \notin E\left(C_{n}^{k}\right)\right\}$, that is, $v_{i}, v_{j}$ and $v_{l}$ induce a $P_{3}$ of $C_{n}^{k}$. We are going to show that $c h_{B}^{\prime}\left(C_{n}^{k}\right)>\max \{2, \alpha\}$, if $k \geq 2, n>4 k$ and $\left\lceil\frac{k}{2}\right\rceil \geq\left(\begin{array}{c}2 \alpha-1 \\ \alpha\end{array}\right)$, by proving that if $H$ is not $\alpha$-choosable then $C_{n}^{k}$ is not $\alpha$-biclique edge-choosable, and that $H$ is not $\alpha$-choosable.

As $n>4 k$ then $C_{n}^{k}$ maximal bicliques are $P_{3}$ and an edge of $H$ is equivalent to a biclique of $C_{n}^{k}$. A vertex coloring of $H$ is an edge coloring of $C_{n}^{k}$ such that the bicliques 
are not monochromatic. Note that by definition of $H$ the degree of any vertex $v_{i j} \in H$ is $d_{G}\left(v_{i, j}\right)=2(\min \{(j-i) \bmod n,(i-j) \bmod n\})$ and $\delta(H)=2$ and $\Delta(H)=2 k$. Since $H$ is not a $K_{1}$, nor an even cycle, nor a $\theta_{2,2,2 c}$, for an integer $c$, then $H$ is not 2-choosable [Rubin et al. 1979] and thus $c h_{B}^{\prime}\left(C_{n}^{k}\right)>2$.

Also note that the resulting graph $H$ has a $K_{\left\lceil\frac{k}{2}\right\rceil,\left\lceil\frac{k}{2}\right\rceil}$ as an induced subgraph. Since a complete bipartite graph $K_{m, m}$ is not $\alpha$-choosable for $m \geq\left(\begin{array}{c}2 \alpha-1 \\ \alpha\end{array}\right)$ [Rubin et al. 1979] then $H$ is not $\alpha$-choosable for $\left\lceil\frac{k}{2}\right\rceil \geq\left(\begin{array}{c}2 \alpha-1 \\ \alpha\end{array}\right)$ and thus $C_{n}^{k}$ is not $\alpha$-biclique edge-choosable for $\left\lceil\frac{k}{2}\right\rceil \geq\left(\begin{array}{c}2 \alpha-1 \\ \alpha\end{array}\right)$.

Corollary 4. The biclique choice index $\operatorname{ch}_{B}^{\prime}\left(P_{n}^{k}\right)>\max \{2, \alpha\}$, if $k \geq 2, n>2 k$ and $\left\lceil\frac{k}{2}\right\rceil \geq\left(\begin{array}{c}2 \alpha-1 \\ \alpha\end{array}\right)$.

Proof. As the power of paths are a specific case of power of cycles then we can use the proof of Theorem 3 to conclude that $c h_{B}^{\prime}\left(P_{n}^{k}\right)>\max \{2, \alpha\}$, if $k \geq 2, n>2 k$ and $\left\lceil\frac{k}{2}\right\rceil \geq\left(\begin{array}{c}2 \alpha-1 \\ \alpha\end{array}\right)$

\section{Conclusion}

In this paper we study the k-biclique-edge coloring choosability problem for some classes of graphs. We prove that $K_{3}$-free graphs are 2-star edge-choosable, chordal bipartite are 2biclique edge-choosable and are not 2-biclique edge-choosable and present a lower bound for the biclique choice index of power of cycles and power of paths. We remark that these last result is not tight. We provide polynomial algorithms to find 2-biclique (star) edgecoloring for $K_{3}$-free graphs and chordal bipartite graphs.

\section{References}

Figueiredo, C. M. H., Filho, H. B. M., Machado, R. C. S., and Dantas, S. (2013). Bicliquecolouring verification complexity and biclique-colouring power graphs. Discrete Applied Mathematics, 192:65-76.

Guedes, A., Ries, B., Sasaki, D., Groshaus, M., Machado, R. C. S., and Dantas, S. (2017). On star and biclique edge-colorings. International Transactions in Operational Research, 24(1-2):339-346.

Kratsch, D. and Kloks, T. (1995). Computing a perfect edge without vertex elimination ordering of a chordal bipartite graph. Information Processing Letters, 55:11-16.

Marx, D. (2011). Complexity of clique coloring and related problems. Theoretical Computer Science, 412:3487-3500.

Rubin, A. L., Taylor, H., and Erdös, P. (1979). Choosability in graphs. Congr. Numer, 26:125-157.

Soulignac, F. J., Groshaus, M., and Terlisky, P. (2012). The star and biclique coloring and choosability problems. Journal of Graph Algorithms and Applications, 18:347-383.

Terlisky, P. (2010). Biclique-coloreo de grafos. Master's thesis, Universidad de Buenos Aires. 